Archives

13 | 1994

Enquêtes

\title{
Une nouvelle du Xv siècle, document d'histoire sociale : il grasso legnaiuolo (« Histoire du gros, menuisier »)
}

\section{Lauro Martines}

\section{OpenEdition}

Journals

Édition électronique

URL : http://journals.openedition.org/ccrh/2712

DOI : $10.4000 /$ ccrh. 2712

ISSN : 1760-7906

Éditeur

Centre de recherches historiques - EHESS

Édition imprimée

Date de publication : 4 octobre 1994

ISSN : 0990-9141

\section{Référence électronique}

Lauro Martines, « Une nouvelle du Xve siècle, document d'histoire sociale : il grasso legnaiuolo ("Histoire du gros, menuisier ») », Les Cahiers du Centre de Recherches Historiques [En ligne], 13 | 1994, mis en ligne le 27 février 2009, consulté le 03 mai 2019. URL : http://journals.openedition.org/ ccrh/2712; DOI : 10.4000/ccrh.2712

Ce document a été généré automatiquement le 3 mai 2019.

Article L.111-1 du Code de la propriété intellectuelle. 


\title{
Une nouvelle $\mathrm{du} X \mathrm{XV}^{e}$ siècle, document d'histoire sociale : il grasso legnaiuolo ( Histoire du gros, menuisier »)
}

\author{
Lauro Martines
}

1 Mon dernier livre présente, traduits en anglais, six contes italiens du Xve siècle ${ }^{1}$. L'édition comprend par ailleurs six études où j'utilise chacun de ces contes comme un texte historique. L'approche est principalement sociale et culturelle, mais l'analyse aborde aussi des problèmes de langue, de style et de métaphore.

Des six contes, quatre viennent de Florence, un de Bologne, le dernier de Sienne ${ }^{2}$. Je les ai choisis parce que chacun illustre à sa manière un aspect important de la vie urbaine à la Renaissance italienne, et un aspect généralement négligé par la documentation habituelle. Ainsi, l'un des récits retenus, celui de Giovanni Gherardi, souligne l'innocence et l'obéissance d'une fille vierge lors de son mariage. Curieusement, toutefois, la fille est quasiment absente du récit, car l'action met en scène les rapports entre sa mère et le gendre de celle-ci. Aussi notre analyse porte-t-elle principalement sur la signification sociale de cette mère, une femme en quelque sorte parfaite, et qui connaît le monde, alors que sa pureté affichée ne nous le laisserait pas prévoir. Face à l'autorité de cette mère et de son propre époux, la fille n'est qu'une pâle comparse.

Oeuvre d'un certain Piero Veneziano (un Florentin en dépit de son nom), une deuxième histoire assez connue, «La Nouvelle de Bianco Alfani », nous entraine dans le monde florentin des années 1420 . Tous les personnages principaux sont réels, et l'on a pu les identifier précisément dans la documentation contemporaine, les archives fiscales du Catasto, par exemple, ou les fonds notariaux anciens ${ }^{3}$. Que l'histoire ait eu lieu relève en partie de la tradition orale; mais nombre de détails narratifs s'accordent parfaitement avec les documents historiques proprement dits.

4 Le personnage-clé, Bianco Alfani, a bel et bien vécu. Né dans une très bonne famille de Florence, c'est néanmoins un bavard, un vantard fanfaron et imbécile, qui va le payer cher et sera cruellement berné par quelques personnes de sa connaissance. Elles lui 
montent avec succès une farce en lui faisant croire qu'il a été élu podestat de Norcia, une petite ville de province située près de Pérouse. S'enorgueillissant de la (fausse) nouvelle, il ne parle plus que de cela et s'en vante partout pour se donner de l'importance; il prépare son départ, embauche comme il y est tenu les gens de sa suite, loue des chevaux, dépensant à tout cela près de 200 florins d'or - une petite fortune -, parce qu'il s'attend à tirer bien davantage de sa charge. Quand finalement il se présente à Norcia avec toute sa suite, la fraude est immédiatement décelée et, comprenant qu'il a été dupé, il en ressent une profonde humiliation. Notre homme se cache donc pendant quelques semaines à la campagne, mais il lui faut bien rentrer à Florence, brisé par la honte et totalement déshonoré. Par tous ses détails, le récit présente le canular (beffa, giarda) comme un événement tout à fait cocasse.

Notre édition comprend aussi un conte du Siennois Gentile Sermini, qui vécut autour de 1430. C'est l'histoire, brutale, d'un paysan appelé Scoppone dans ses rapports avec son maitre Bartolomeo Bonsignori, un noble siennois. Laid, avare, sale, égoïste, rusé et totalement dépourvu du respect dû à son seigneur naturel, Scoppone est une tache, une verrue sur la campagne siennoise. Un beau jour, le noble Bonsignori trouve le moyen de le métamorphoser, non sans qu'une bande de jeunes nobles ait au préalable passé à tabac le paysan, en mimant comiquement les rituels de l'administration judiciaire. Scoppone deviendra un homme nouveau. Sur les instances de son bon seigneur - Bonsignori - il sera même rebaptisé. L'histoire met en lumière les relations entre ville et campagne, citadin et paysan, propriétaire et métayer, noble et roturier, l'idéal recherché face à une réalité peu satisfaisante. Ajoutons que le monde rural est vu à travers le seul filtre de la classe dirigeante citadine.

6 Le livre inclut aussi le seul conte qu'ait écrit Laurent le Magnifique, et celui d'un écrivain bolonais, Giovanni Sabbadino degli Arienti. Le premier, qui narre une séduction par duperie, exprime clairement la rivalité entre Florence et Sienne et leur aversion réciproque. Le second porte sur la beauté d'un jeune prêtre travesti et montre l'ambiguïté profonde des attitudes sociales à l'égard de l'homosexualité.

7 Venons-en maintenant à «La Nouvelle du Gros menuisier", dont la version la plus complète est celle d'Antonio di Tuccio Manetti (v. 1423-1497).

8 Un menuisier surnommé «il Grasso » (« le Gros ») fait partie d'une joyeuse bande qui se réunit souvent pour dîner. Le Gros, un soir, manque la fête, et ses compagnons décident d'en tirer vengeance. Filippo Brunelleschi, qui est des leurs, suggère de lui jouer une farce en lui faisant croire qu'il est devenu un autre. Lui-même lance le jeu en s'enfermant dans la maison vide du Grasso, et, quand ce dernier veut rentrer chez lui, il contrefait sa voix et, à travers la porte close, s'adresse à lui comme s'il était un certain Matteo. Le pseudoMatteo, tout éberlué, est alors arrêté par des sbires pour les dettes du véritable Matteo et jeté en prison. Il s'y convainc qu'il est devenu Matteo, quand non seulement ses codétenus, parmi lesquels un juge compatissant, l'admettent sous sa nouvelle identité, mais des passants de sa connaissance feignent de le reconnaître comme Matteo. Après une journée difficile passée là sans boire ni manger, il est tiré de sa geôle par les vrais frères de Matteo, qui l'emmènent chez eux, le font admonester par le prêtre de la paroisse pour le convaincre d'accepter définitivement son identité nouvelle, puis le droguent et le reportent endormi dans son lit. A son réveil, éberlué d'être à nouveau reconnu comme le Grasso même par les frères de Matteo, il erre dans la cathédrale où il rencontre le maître d'oeuvre de la supercherie, Brunelleschi, qui, accompagné de Donatello puis de Matteo, achève de le troubler en lui rapportant l'aventure symétrique 
prétendument vécue par le vrai Matteo qui se serait pris pour le Gros pendant tout le temps où ce dernier était pris pour lui. Matteo en personne, qui est bien entendu de mèche, confirme de mille détails l'histoire qu'il aurait de son côté rêvée, plongeant le malheureux menuisier dans une douloureuse incertitude sur le rêve et la réalité des derniers jours. Il lui faudra boire jusqu'à la lie la coupe de l'humiliation lorsque, le lendemain, il comprendra qu'il a été l'objet d'une méchante farce, et qui en est l'auteur. Décidant sur un coup de tête de quitter Florence, il ira jusqu'en Hongrie exercer ses talents et ne reviendra dans sa patrie que des années plus tard.

J'ai retenu ce récit parce qu'il me troubla beaucoup, moi historien, lorsque je le lus pour la première fois. Je fus alors frappé par toute une série de questions, qui sont d'ordre purement historique. En voici quelques unes.

10 1) Pourquoi aucun historien n'a-t-il jamais analysé cette histoire de façon systématique ? André Rochon, qui a beaucoup écrit sur elle, est un spécialiste d'histoire littéraire ${ }^{4}$.

11 2) Le problème de l'identité individuelle n'a-t-il jamais été posé en tant que problème historique?

3) L'histoire ne comporte-t-elle pas une intrigue relevant des relations sociales quotidiennes?

13 4) S'agit-il d'une farce qui a vraiment eu lieu? Tous les personnages, comme dans l'histoire de «Bianco Alfani », ont réellement vécu.

14 5) Si l'histoire se fonde sur un événement réel, doit-on considérer le menuisier Grasso tout simplement comme un idiot? Ou bien la vie quotidienne à Florence pouvait-elle déboucher sur des situations proches de celles de ce récit? De fait, le Grasso du conte ne m'apparaissait ni comme un crétin ni comme un fou.

6) Comment expliquer que les amis du Grasso, tous ses amis, aient considéré leur supercherie comme une plaisanterie à crever de rire ? Pourquoi ce sens de l'humour dans la Florence du Quattrocento ? Quels en étaient les fondements sociaux et culturels?

7) Pourquoi l'histoire du Grasso a-t-elle commencé à perdre de son sel au cours du XIX siècle?

17 8) Comment les amis du Grasso ont-ils pu assez contrôler les rues de leur ville pour réussir si parfaitement leur canular?

9) Qu'est-ce qui, dans le statut social du Grasso, a pu faire de lui la cible d'une telle farce?

19 10) Pourquoi le Grasso, dans la crise qu'il traverse, ne semble-t-il jamais se poser de questions religieuses, ni même penser à demander secours au curé de sa paroisse?

Et il est bien d'autres questions encore que je poserai par la suite ${ }^{5}$.

21 Il existe au moins quatorze manuscrits différents, et cinq versions de « La nouvelle du Grasso » qui tous appartiennent au Xve siècle ${ }^{6}$. Ajoutons que l'auteur de la version la plus importante, Manetti, affirme dans sa biographie de Brunelleschi que la supercherie inspirée par le célèbre artiste pour accabler le menuisier fut réellement mise en oeuvre. En bref, nous possédons plus de témoignages sur la véracité de l'histoire que sur bien d'autres événements historiques!

22 Outre le nombre des manuscrits et la force de la tradition orale, nombre de détails de l'histoire, ainsi que certaine tournure d'esprit inspirant la vie sociale florentine me convainquent que Grasso fut sûrement la victime de son excellent ami Filippo Brunelleschi. 

disent beaucoup. Chez Austen, on comprend dès les premières lignes la condition financière de la jeune femme ou de la famille qui seront au coeur de l'action. Chez les conteurs italiens de la Renaissance, et cela d'autant plus qu'ils restent proches de la tradition orale, le lecteur est immédiatement averti du statut social des personnages. Il est clair que, dans la pratique, le conteur était conscient de la nécessité de définir d'emblée la condition de ses personnages: question centrale s'il voulait en faire comprendre le comportement, et les conséquences de l'action. Du quatorzième au seizième siècle, les écrivains se sont tous nettement employés à ce cadrage.

24 joyeuse bande rassemblée autour de Tommaso Pecori, Filippo Brunelleschi, Giovanni di Francesco Rucellai et Donatello, soit quelques uns des principaux conjurés contre le gros menuisier. Leur « brigade » comprend des membres de l'oligarchie, des marchands aussi, et des artisans pratiquant un type de travail manuel exigeant de l'ingegno, c'est-à-dire une certaine intelligence, de l'imagination, peut-être de la fantaisie. Dans l'Europe du début du Xve siècle, c'est une association rare, je dirais même unique. De tels cercles regroupant artisans et membres de l'oligarchie pour dîner assez régulièrement ensemble, ne se retrouvent alors ni à Venise, ni à Milan, ni à Rome. Tout au plus en rencontrerait-on à Sienne, encore que cela me semble peu probable. A Florence, cette joyeuse compagnie au moins témoigne d'une ouverture sociale remarquable. Est-ce là précisément la raison du soupçon de nervosité ou de tension qui touche quelques uns de ses membres? Car il ne s'agit absolument pas d'une société d'égaux : ils reconnaissent leurs différences sociales, et rendent certainement honneur au rang élevé de certains d'entre eux. Le Grasso est luimême client de son "ami» Giovanni Rucellai. Quand, en prison, ils ont une brève conversation où Giovanni feint de ne pas reconnaître le Grasso, il dit de celui-ci : « Egli è tutto mio » (« il m'est tout dévoué ») . Giovanni entend par là que c'est un homme qui lui est fidèle, qui est son fidèle, à lui un gentilhomme qui peut s'attendre à être entouré de « fidèles ».

On peut se demander, de surcroît, pourquoi Rucellai tutoie un homme qu'il feint de n'avoir jamais vu, alors que le Grasso le vouvoie. Giovanni assurément considère l'allure du gros menuisier et, à ses vêtements, le classe aussitôt comme un artisan, un travailleur manuel : il lui donne donc du «tu », ce qu'il n'aurait pas fait avec le juge emprisonné. En revanche, le Grasso, présumé inconnu, à la vue d'un gentleman emploie d'emblée le pronom "vous »; plus tard, lorsqu'il engagera la conversation avec le juge, il recourra aussi au vouvoiement, bien que le juge le tutoie. Bref, dans la nouvelle du Grasso, l'usage des pronoms respecte les différences sociales.

Autre trait à noter : après le dénouement de la supercherie, le menuisier peut quitter Florence sans se préoccuper de ses propriétés foncières, tout simplement parce qu'il n'en possède pas. Pour tout bien il a ses outils de travail, dont il emporte l'essentiel en Hongrie. Ce qu'il laisse dans l'atelier, que d'évidence il loue, fait partie de la dot de sa mère ou servira à la garantir.

27 Tout me porte à croire, enfin, que le Grasso, comme peut-être Donatello, était le plus modeste de la compagnie des dîneurs. Le conte même nous en assure: «ils étaient presque tous d'une condition supérieure à la sienne $»^{8}$.

Se sentant «abaissés » (scornati, je dirais « snubbed » en anglais) lorsque Grasso ne vient pas souper avec eux, les membres de la compagnie décident de «se venger de cette 
injure ». Il s'agit donc là d'une histoire de différence, de punition et de peine sociales, visant à enseigner au Grasso le respect envers ses supérieurs.

Dans l'esquisse sociale des premières pages du récit, seul le Grasso porte un sobriquet et est présenté comme un homme travaillant de ses mains. Par le qualificatif «ser» marquant son patronyme ${ }^{9}$, au contraire, Filippo di ser Brunellesco - « maraviglioso ingegno ed intelletto » («personnage d'une nature et d'une intelligence admirables »-est associé au monde du latin et de l'érudition. Hormis Donatello, le menuisier est d'ailleurs fort probablement le plus jeune de la bande, et par suite l'un des plus vulnérables, la cible potentielle d'une méchante farce. En outre, il est grand, gros, gauche, fantasque, peutêtre un peu timide face à des gens comme Pecori et Rucellai, un tantinet simplet : simplet par rapport au monde très dur et très pratique de Florence, où l'apparence rêveuse est vite interprétée comme simplicité d'esprit. Je croirais volontiers, d'ailleurs, qu'en dépit de ses apparences et de sa modeste condition financière, Grasso avait curieusement quelque chose du parvenu; son père et son grand-père, en effet, avaient été prieurs, autrement dit l'un et l'autre avaient jadis fait partie du petit conseil (la Signoria) qui gouvernait la ville; à Florence, tous les gens bien le savaient certainement.

Tels étaient les traits qui rendaient Grasso particulièrement vulnérable aux supercheries du cercle Pecori-Brunelleschi. Soulignons aussi qu'en dehors de sa mère, qui se trouve hors de la ville pendant la farce, le menuisier semble dépourvu de parenté proche et par conséquent tout à fait isolé : une faiblesse qui peut se révéler pénible dans les villes italiennes du Quattrocento. Les conjurés doivent en effet une bonne part de leur succès à l'isolement du Grasso. Personne ne le cherche ; quand il est en prison, bien que quelques comparses le connaissent de vue, personne n'en sait le nom, et lui, ainsi relégué et désorienté, ne peut se rappeler un seul parent pour l'aider. Et bien entendu, il ne peut faire appel à ses prétendus amis auxquels il craindrait trop de révéler l'état mental auquel il est réduit.

31 Le Grasso est arrêté à la tombée de la nuit, à l'heure du dîner, afin que ses voisins ne s'en avisent pas. Le lendemain, ses prétendus « frères » - c'est-à-dire les frères de Matteo son alter ego - le font sortir de prison derechef le soir, à la nuit tombée, et il n'y a de nouveau presque aucune chance qu'un passant le reconnaisse comme le Grasso tandis qu'ils franchissent le Ponte Vecchio.

32 Ainsi, les amis du Grasso réussissent à l'isoler complètement tout en l'enserrant dans un réseau de fausses relations sociales. Autrement dit, ils contrefont Florence ; ils deviennent Florence; ils entourent si bien le pauvre artisan que celui-ci commence à croire que les gens de Florence, tous les Florentins, le reconnaissent comme Matteo. Telle est l'astuce de Brunelleschi et ses complices. Pendant le complot, ils font intervenir près de vingt personnes, sans compter le juge et les autres prisonniers. A la fin de l'histoire, le juge luimême avoue que n'importe qui aurait trouvé la farce « fort malaisée à déjouer ", tant fut grande la malice de Brunelleschi.

Je voudrais maintenant soulever la question de la vie privée dans ses rapports avec la vie publique à Florence pendant la Renaissance.

Le Grasso a son atelier sur l'une des places centrales de la ville, la place du baptistère San Giovanni, qui est le saint patron de Florence. Son atelier est donc un endroit où passe beaucoup de monde, et les grands travaux à la cathédrale toute proche alimentent aussi ce va-et-vient. Notre menuisier, en outre, a fait son apprentissage dans un atelier proche du Marché Neuf, autre coeur de la ville. Par suite, tout ce qu'il est, tout ce qu'il accomplit 
dans un métier dont il est un spécialiste assez connu, est étroitement lié à la reconnaissance d'autrui, à la reconnaissance du travail de ses mains. Le Gros avait, semble-t-il, pour nom de famille Ammannatini, mais le conte n'en fait jamais mention, et tout le monde connaît notre personnage comme « il Grasso legnaiuolo ». Sa famille, je l'ai dit, est inexistante, au moins dans ce récit. Lui-même ne possède en propre aucun bien immobilier; enfin, c'est un célibataire. Bref, il incarne tout le contraire de ce que représente le fils d'une bonne famille florentine. Aussi, si ce monde dans lequel il se reconnaît dans la mesure où il en est reconnu se retourne contre lui et proclame de façon insistante qu'il est Matteo et non pas le Grasso, que peut-il faire d'autre sinon, en bon conformiste, y croire? C'est ainsi que par un jeu malin d'assonances Manetto Ammannatini (dit "le Gros") devient Matteo Mannini.

A l'évidence c'est un conformiste. Toujours respectueux et bien élevé, quand, à la suite de son bref entretien avec Giovanni Rucellai, il est au bord des larmes, il avoue avoir toujours su se garder des dettes, des situations difficiles (zacchere), "ainsi que de cent autres erreurs qui peuvent causer des embarras ». Bien que dépourvu d'un nom aussi illustre que Rucellai, il redoute infiniment les commérages.

Ce qui revient tout simplement à dire que, puisque l'identité du Grasso se construit, se confirme et prend sa valeur dans un espace public, l'homme ne peut que s'effondrer lorsque tous ceux qui habitent cet espace, par un changement aussi effroyable que mystérieux, le reconnaissent soudain comme Matteo.

Pour cet artisan, la société florentine est une sorte de miroir ${ }^{10}$; si son image s'y change, si les réactions des membres de cette société en révèlent la mutation, il lui faut alors changer lui aussi et se conformer à cette image, au risque, sinon, d'être pris pour un fou. C'est donc lui-même qui va provoquer l'un des épisodes qu'il ressentira le plus durement, la conversation dans la cathédrale avec Brunelleschi et ses complices. Ce matin-là, en effet, tout inquiet à la suite de sa rencontre avec les frères de Matteo, il décide de visiter le Duomo. Il veut réfléchir ; il entend une fois pour toutes établir son identité. Il aurait été bien naturel que ce désir l'amenât à rentrer chez lui et à s'y abandonner à ses pensées, seul, sans être dérangé. Mais non: il est poussé par l'urgence de confirmer, voire de rétablir son identité, et cela ne peut se faire que dans le regard de ses voisins, des gens de sa paroisse, de ses patrons, bref de la communauté florentine. Il est normal qu'il se rende alors à Santa Maria del Fiore, l'un des espaces les plus publics de la cité, et il y va " pour réfléchir à son cas et mieux éclaircir (per certificarsi meglio), grâce aux rencontres qu'il ferait, s'il était le Grasso ou Matteo $»^{11}$.

Venons-en maintenant aux conjurés. Sont-ils contrairement au Grasso, des individus uniques, peu modelés par la société environnante? Considérons-les dans leurs rapports avec le menuisier. Ce sont tous d'excellents acteurs, ils sont parfaitement organisés, concentrés sur le même but : ils sont soudés en une équipe. Matteo et ses frères, ainsi que Giovanni Rucellai, jouent superbement leur rôle. Même le juge, lorsqu'il comprend qu'il s'agit d'une farce, entre immédiatement dans le jeu des mystificateurs. Attitude exceptionnelle, qui, si l'on y pense, ne peut paraître normale. Comment l'expliquer?

Il me semble évident que l'empressement général à jouer comme au théâtre relève du très vif sentiment d'appartenance à une communauté restreinte que tous éprouvent; la participation collective au groupe va de soi, surtout quand il s'agit de prendre quelqu'un au piège, plus encore quand la victime désignée révèle en quoi que ce soit un brin de nonconformisme. Car la vie sociale florentine est durement conformiste, et ce trait explique la popularité et la fréquence des farces et canulars (beffa, natta, giarda) dans la vie 
quotidienne et la littérature de la Renaissance. Il s'agit non seulement de s'y montrer plus malin que le voisin, mais de chasser l'autre du troupeau. L'esprit conformiste de la participation se concrétise chez les farceurs : en travaillant ensemble et coopérant, ils se transforment plus encore en acteurs. Ils marginalisent leur victime plus efficacement en mettant à nu sa sottise, sa vanité, sa bizarrerie, ou toute autre particularité l'écartant de la norme. C'est dire que la beffa allie deux aspects dominants de la vie sociale florentine : ses tendances à la concurrence agressive, que Ronald Weissman a fort bien analysée il y a quelques années ${ }^{12}$, et son côté conformiste. Dans la beffa, l'esprit concurrentiel de la vie citadine est mis au service de ses exigences conformistes.

Le Grasso était un conformiste consciencieux, mais il avait aussi un air un peu excentrique, assez excentrique, en tous cas, pour s'attirer les rires vengeurs de son groupe. C'est ainsi que nous en revenons à l'une de nos premières questions : comment se fait-il que les farceurs, tous amis de Grasso, à l'exception de Matteo et ses frères, aient pu lui faire une plaisanterie aussi méchante en la considérant comme une merveille de drôlerie ? La raison en est que la vie cruellement conformiste de Florence imposait aux voisins l'arme pédagogique du ridicule, une arme employée contre quiconque osait sortir du commun, du fait de son amour-propre, de sa bêtise, ou de quelque bizarrerie personnelle. Plus on humiliait la victime, et plus efficace était la sanction sociale, plus fort le rire, d'autant que la punition avait une visée clairement morale.

Pourquoi, dès lors, ne pas rire aux éclats, en toute bonne conscience?

\section{NOTES}

1. An Italian Renaissance Sextet: Six Tales in Historical Context, New York, 1994.

2. Il s'agit de : 1) Giovanni Gherardi da Prato, Novella di madonna Ricciarda ; 2) Gentile Sermini, Bartolomeo Bonsignori; 3) Giovanni Sabadino degli Arienti, Certi gioveni da Arezio; 4) Piero Veneziano, Novella del Bianco Alfani; 5) Lorenzo de' Medici, Novella di Giacoppo; et 6) Antonio Manetti, Novella del Grasso Legnaiulo. Les nouvelles 4, 5, 6 ont été traduites dans le récent volume de la Pléiade, Conteurs italiens de la Renaissance, éd. sous la dir. d'Anne Motte-Gillet, Gallimard, 1993 (respectivement aux pages 51-65 pour la quatrième, 1227-36 pour la cinquième et 181-214 pour la sixième).

3. Vittorio Rossi, "Sulla novella del Bianco Alfani", dans Scritti di critica letteraria, 3 vol., Florence, 1930, II, p. 371-400.

4. A. Rochon, «Une date importante dans l'histoire de la beffa: La nouvelle du Grasso Legnaiuolo », dans Formes et significations de la « beffa » dans la littérature italienne de la Renaissance (Deuxième série), Paris, 1975.

5. Pour certaines d'entre elles, je renvoie le lecteur au chapitre que j'ai consacré à l'histoire du Grasso dans mon récent livre An italian Renaissance Sextet

6. Cf l'édition de Domenico de Robertis et Giuliano Tanturlo, Vita di Filippo Brunelleschi preceduta da la Novella del Grasso, Milan, 1976

7. Cf. la traduction de la Pléiade, citée supra, p. 187. 
8. Generalmente crano quasi tutti di migliore qualità e condizione di lui [il Grasso]; ibidem, p. 182. Le manuscrit Palatino 200 fait à peu près la même remarque : «Era di bassa condizione a rispetto degli altri ».

9. «Ser » est le titre porté en Toscane par les notaires en particulier.

10. Cf An Italian Renaissance Sextet, p. 224-5

11. Trad. de la Pléiade, p. 201.

12. Ritual Brotherhood in Renaissance Florence, New York, 1982. 